Doctrina

DOI: $\underline{\text { https://doi.org/10.22235/rd.vi20.1850 }}$

\title{
"Third Party Funding"; su relevancia e influencia actual en el mundo del arbitraje internacional
}

Third Party Funding, its relevance and current influence in the world of international arbitration ${ }^{1}$

"Third Party Funding"; sua relevância e influência atual no mundo da arbitragem internacional

\author{
Santiago Theoduloz ${ }^{1}$ \\ ${ }^{1}$ Universidad de la República \\ ORCID: https://orcid.org/0000-0002-3316-5712
}

Para citación: Theoduloz, Santiago, "Third Party Funding"; su relevancia e influencia actual en el mundo del arbitraje internacional,

DOI: https://doi.org/10.22235/rd.vi20.1850 en Revista de Derecho $\mathrm{N}^{\circ} 20$ (dic. 2019): 159-187. ISSN 1510-3714, ISSN On line 2393-6193, 15-28

\section{Resumen:}

El presente trabajo tiene como objeto analizar el fenómeno de los Third Party Funding o terceros financistas y su influencia y desarrollo actual en el mundo del arbitraje internacional comercial y de inversión. En base a ello se analizan los principales aspectos y características del fenómeno y las distintas regulaciones existentes en el mundo actual. Asimismo, se estudia como el fenómeno puede influenciar en los estándares de independencia e imparcialidad de los árbitros y como consecuencia de ello en la eventual existencia de conflictos de intereses.

Palabras clave: financiamiento por terceros, arbitraje, arbitraje internacional, arbitraje comercial, arbitraje de inversión, conflictos de intereses, independencia e imparcialidad.

\section{Abstract:}

Recibido: 18/03/19. Evaluado y corregido: 15/7/19. Aceptado: 29/7/19 
The main object of this work is to analyse the phenomenon of Third Party Funding and its influence and development in the current commercial and investment arbitration world. Thus, the work analyse the main aspects and characteristics of the TPF and the different regulations that already exists. In addition, the work studies how the use of TPF could influence in the independence and impartiality of the arbitrators and therefore, the possibility of the existence of conflict of interests.

Keywords: Third Party Funding, arbitration, international arbitration, commercial arbitration, investment arbitration, conflict of interest, independence and impartiality.

\section{Resumo:}

Este artigo tem como objetivo analisar o fenômeno do financiamento de terceiros ou financiadores terceirizados e sua atual influência e desenvolvimento no mundo da arbitragem comercial e de investimento internacional. Com base nisso, analisam-se os principais aspectos e características do fenômeno e as diferentes regulamentações no mundo de hoje. Também é estudado como o fenômeno pode influenciar os padrões de independência e imparcialidade dos árbitros e como resultado da possível existência de conflitos de interesse.

Palavras-chave: financiamento terceirizado, arbitragem, arbitragem internacional, arbitragem comercial, arbitragem de investimento, conflitos de interesse, independência e imparcialidade.

Sumario: I. Introducción. II. El concepto y origen de los TPF. 1) El concepto de TPF. 2) El origen y evolución de los TPF. 3) La regulación actual de los TPF. III. Conflictos de intereses: El gran desafío de los TPF. 1) La supervivencia y desarrollo de los TPF y la influencia de los aspectos éticos. 2)Conflictos de intereses y situaciones de riesgo ante la utilización de los TPF. 3)La obligación de revelar la existencia de un TPF. 4) ¿Por qué preocupa conocer la existencia de un TPF? 5) ¿Qué elementos y a quién revelar la existencia del TPF? IV. Conflictos de intereses: ¿Puede el uso de TPF suponer un crecimiento en materia de conflictos de intereses? V. Consideraciones Finales. Bibliografía.

\section{Introducción}


El mundo del arbitraje internacional se ha visto influenciado en los últimos años con la aparición de una nueva figura como es el "Third Party Funding" (en adelante por sus siglas en inglés como "TPF" o como "financiamiento"”) que en español puede traducirse como "Terceros Financistas", "Inversores en Pleitos" o "Financiamiento por Terceros". Dado el origen de la figura en idioma inglés así lo seguiremos referenciando en el presente artículo.

Los TPF pueden definirse como terceras partes, ajenas al proceso arbitral, cuya función es financiar a una o ambas partes lo costos de un proceso arbitral a cambio de un beneficio. Decimos beneficio, puesto que como analizaremos en el presente artículo, en nuestra postura aun en el caso de que estos financistas no persigan un beneficio económico también se los puede considerar tales.

La figura de los TPF no es nueva, pero si es nuevo su uso y desarrollo en el mundo del arbitraje. En este sentido, esta forma de financiamiento de disputas tiene origen en el área de litigios judiciales, donde en distintas jurisdicciones se vio afectado por diferentes prohibiciones. Naturalmente dichas prohibiciones tenían un fundamento principalmente ético y en como la figura de financistas y los beneficios económicos que ellos reportan, podían suponer en el caso la utilización o creación de litigios verdaderamente inexistentes como forma de buscar un beneficio económico.

No obstante, el ingreso de este nuevo actor al mundo del arbitraje ha traído consigo diversas discusiones que se centran en diversos elementos de contenido principalmente ético. Las mismas se han cristalizado en dos planos (i) la influencia que pueden tener en la imparcialidad e independencia de los árbitros en sus decisiones y (ii) cómo este elemento debería influenciar al determinar los "security for costs" por parte de un determinado tribunal arbitral. En el presente, nos centraremos únicamente en el primer aspecto.

Ahora, ¿es legítima la utilización de estos financistas? En Latinoamérica la respuesta afirmativa se impone. No obstante, la respuesta no es unánime en el mundo entero. En este sentido, en varios países del sistema anglosajón -en el cual el TPF nació- han existido y existen prohibiciones para su utilización. Dichas prohibiciones surgen con anterioridad a la posibilidad de su utilización en el arbitraje y tienen su fundamento principalmente en la posibilidad de que produjeran conflictos de naturaleza ética en el ámbito judicial. Con carácter general, las respectivas prohibiciones han cedido para dar paso a la utilización 
de los TPF, sin desconocer que aún sigue siendo prohibido en determinadas jurisdicciones.

En Latinoamérica, países de tradición civilista, no existe prohibiciones, pero tampoco existe una regulación del fenómeno al menos desde el punto de vista legislativo. ${ }^{2}$ Por ello, en tanto el principio de que todo lo que no está prohibido, está permitido ${ }^{3}$, la conclusión que se impone prima facie es que, ante la inexistencia de dicha prohibición y regulación, los TPF se encuentran claramente permitido en las jurisdicciones latinoamericanas. Igualmente, esto no significa que pueda efectuarse su utilización sin determinados límites y que determinados estándares éticos, así como la incorporación de determinadas reglas a los procesos arbitrales, puedan igualmente influenciar en la regulación de los TPF. Uno de los principales elementos a considerar a la hora de pactar una clausula arbitral es la conveniencia de someter la disputa a un arbitraje ante los costos que un eventual proceso arbitral puede suponer para las partes. Conocer de antemano la posibilidad de contar con un respaldo de un TPF puede ser completamente beneficioso para las partes al momento de contratar. Tanto en Uruguay $^{4}$ como en Argentina ${ }^{5}$, hace muy poco se aprobaron las leyes que regulan el arbitraje comercial internacional, lo que augura, sin hesitaciones, el crecimiento de un mercado, donde elementos como los TPF deben ser analizados. Ello dado que sin lugar a duda su desarrollo va a influir inmediatamente en el medio local, flexibilizando un elemento relevante para el arbitraje como es el costo que el proceso implica.

Naturalmente, el estudio de los TPF ha puesto de relieve algunos aspectos que en principio podrían considerarse negativos, aspectos que han traído consigo ciertos detractores. Estos aspectos se centran en visualizar la existencia de determinados conflictos de intereses que pueden influir en los árbitros al tomar decisiones, así como quienes les preocupa la creación de una industria que genere más litigios por los

\footnotetext{
${ }^{2}$ Solamente existe una referencia al fenómeno en Brasil desarrollada por parte de la Centro de Arbitraje y Mediación de la Cámara de Comercio Brasil Canadá (CAMCCBC).

${ }^{3}$ Esta referencia alude principalmente a la inexistencia de reglas (disposiciones normativas) que prohíban los TPF. Por lo tanto, se trataría de una permisión débil: inexistencia de reglas que prohíban la intervención de los TPF.

${ }^{4}$ Ley 19.636, Publicación 26 de julio de 2018, Poder Legislativo, Uruguay.

${ }^{5}$ Ley 27.449, Publicación 26/7/2018, Congreso Argentino.
} 
beneficios que los mismos puede reputarles a las partes y a estos terceros. Por el contrario, en el presente artículo se demostrará cómo un buen estudio del tema y la regulación de algunos aspectos relevantes en la materia suponen un riesgo muy menor e invitan al desarrollo y promoción de los TPF.

Uno de los elementos más relevantes a analizar es la obligación que tienen o deberían tener las partes de revelar la existencia de un TPF. Lo que supone en su caso determinar cual sería el alcance de los elementos a revelar y si en su caso ello verdaderamente supone el fin de toda amenaza al estándar de imparcialidad e independencia que debe caracterizar a todo árbitro.

En el presente trabajo analizaremos en primer lugar los orígenes de los TPF, así como su concepto. Luego, efectuaremos algunas precisiones respecto a los distintos regímenes que regulan el fenómeno. De allí, pasaremos a analizar aquellos aspectos relativos a los TPF y los respectivos conflictos de intereses y por último algunas referencias de como el fenómeno se está desarrollando y si esto puede suponer en el caso que se generen situaciones de conflicto de intereses.

Nos encontramos ante un fenómeno nuevo, que debe ser estudiado y al cual le encontramos un potencial futuro que hacen de vital interés conocer el mismo. Es un fenómeno que además puede afectar conceptos centrales del arbitraje como son la imparcialidad e independencia de los árbitros por lo que una mala utilización de este puede incluso afectar al arbitraje como mecanismo de solución de conflictos generando un importante impacto en su desarrollo futuro.

\section{El concepto y origen de los TPF}

\section{El concepto de TPF}

Cuando hablamos de los TPF nos estamos refiriendo a una tercera parte, ajena a las propias partes del proceso arbitral que financian al demandante o al demandado sometidos bajo un proceso arbitral y en busca de un beneficio. No obstante ello, existen algunas discusiones sobre que comprende el concepto y cuando nos encontramos ante un verdadero TPF. 
Los TPF pueden estar presentes tanto en casos de arbitraje comercial como en arbitrajes de inversión y por tanto no pueden quedar excluidos a un solo tipo de ellos. Siendo las conclusiones que se puedan adoptar en el presente aplicables a ambos tipos de arbitraje. A los efectos de definir un TPF debemos visualizar si se encuentran presentes o no determinados elementos que hacen al concepto en sí. Así, vamos a estar frente a un TPF cuando; (i) Un tercero ajeno a las partes; (ii) soporte parte o la totalidad de los gastos (costos y honorarios) de un arbitraje, asumiendo en definitiva los riesgos de la actividad arbitral; (iii) con un determinado interés en la disputa que le reportará un beneficio.

El elemento "riesgo" es vital, puesto que es el propio financista que toma el riesgo que la parte financiada no quiere o no puede adoptar. En referencia a este último punto, dado los riesgos que supone el estar bajo un proceso arbitral, determinada parte del proceso arbitral puede querer invertir en otra necesidad empresarial o de cualquier otra naturaleza y transferir el riesgo que el proceso arbitral supone al TPF.

Ahora, esta transferencia del riesgo puede tener distinta causa, y en ningún caso puede significar -como se ha sugerido- que la parte en cuestión no pueda asumir los riesgos de la actividad arbitral. Así, en el caso Manuel Garcia Armas y otros v. Venezuela, el Tribunal sostuvo ser consciente que:

(...) la existencia de financiamiento por terceros no constituye per se prueba de la insolvencia de aquellos que recurren a dicho financiamiento. Ello porque, como remarcan los Demandantes, los motivos por los cuales se recurre a financiamiento por terceros pueden ser muy variados, incluyendo simples decisiones comerciales, mayor distribución de riesgos, razones de flujo de caja y otro tipo de motivos que nada tienen que ver con la insolvencia. Pero, naturalmente, dentro de ese abanico de opciones la insolvencia o dificultades financieras son indudablemente algunos de los posibles (y frecuentes) motivos para acudir a financiamiento por terceros. ${ }^{6}$

Una de las razones por la cual se han encontrado detractores de los TPF se basan en el hecho de que atento al análisis financiero que hace un TPF respecto del caso, el conocimiento de este hecho por parte del Tribunal arbitral haría considerar que la parte financiada es quien tiene en definitiva la "razón" en el conflicto planteado. Elemento que ha sido rechazados por aquellos que trabajan en el área. Es verdad que existe un análisis

\footnotetext{
${ }^{6}$ Manuel García Armas y otros v. Venezuela. 20 de junio de 2018. Caso CPA- Corte Permanente de Arbitraje. $N^{\circ} 2016-08$
} 
de riesgo, pero este análisis presenta una infinidad de variables donde también el rechazo de una determinada pretensión es en su caso considerados.

Utilizamos el vocablo "interés" al definir a los TPF en tanto entendemos es un concepto más amplio que por ejemplo "beneficio económico". Así en nuestro concepto -sin perjuicio de ser un elemento por demás discutido doctrinariamente- entendemos que el interés del financista puede ser de tipo económico o no. Por tanto, en nuestra concepción, aquellos fondos que no tienen un fin económico también deben ser considerados como TPF y volcarles las conclusiones referidas en el presente artículo.

De esta forma, el desarrollo de este fenómeno ha traído consigo nuevos desafíos que también corresponde estudiar, como es el caso de los fondos que actúan sin fines de lucro. Es decir, fondos que persiguen un beneficio pero que no es económico. Ahí, encontramos uno de los principales ejemplos en el caso de Uruguay quien tuvo el apoyo de un financista para su defensa en el caso que iniciara oportunamente la tabacalera Philips Morris ${ }^{7}$.

Por su parte, en su definición, el reciente "Acuerdo Económico y Comercial Global entre Canadá y la Unión Europea (en adelante “AECG entre Canadá y la Unión Europea”), incluye claramente a los TPF sin fines de lucro al referir en su art. 8.26 a los términos “donación" o "subvención"8

Ciertas definiciones al referir al concepto de TPF hablan de que la actividad debe ser ejercido por una persona profesional ${ }^{9}$ en el área (entendida como alguien que debe ejercer

\footnotetext{
${ }^{7}$ Phillips Morris vs. Uruguay. 8 de Julio de 2016 (envío a las partes), Caso CIADICentro Internacional de Arreglo de Diferencias Relativas a Inversiones. CN. ARB/10/7. ${ }^{8}$ El tratado en su versión en inglés manifiesta: "The disclosure shall be made at the time of the submission of a claim, or, if the financing agreement is concluded or the donation or grant is made after the submission of a claim, without delay as soon as the agreement is concluded or the donation or grant is made." Acuerdo Económico y Comercial Global entre Canadá y la Unión Europea (Art. 8.26). Acceso el 4 de marzo de 2019.

http://ec.europa.eu/trade/policy/in-focus/ceta/ceta-chapter-by-chapter/ 9 “... (a) a professional person or entity which (b) finances a legal claim or defence in which it has no pre-existing interest, and (c) from which it receives a financial benefit...” en Derric, Yeoh. 2016: "Third Party Funding in International Arbitration: A Slippery Slope or Levelling the Playing Field?” Journal of International Arbitration.
} 
dicha actividad en forma habitual). Discrepamos de dicha definición dado que entendemos que cualquier persona o empresa (asegurador, estudio de abogado, etc.) que cumpla con las características de un TPF debe ser considerado como tal.

En definitiva, en nuestra concepción, un TPF se define como una tercera parte ajena al proceso arbitral, la cual no tiene un interés previo en el litigio y que financia los costos del arbitraje ya sean los que incurre el demandante o demandado en búsqueda de un beneficio que puede ser económico o no.

\section{2) El origen y evolución de los TPF}

En la actualidad el fenómeno de los TPF ha crecido enormemente en la materia arbitral y es uno de los aspectos que mayormente se discute en el ámbito del arbitraje. No obstante, su origen es en la materia de litigios, es decir en asuntos que proceden ante la justicia ordinaria. Es el propio derecho anglosajón donde se desarrollaron las teorías de "champerty and maintenance". Ambas doctrinas que tienen una relevante influencia hasta la fecha suponen exactamente una prohibición para el uso de cualquier tipo de financiamiento de las partes sometidas a un juicio. Como fundamento en dichas doctrinas y durante todo el siglo XIX el financiamiento de litigios fue considerado ilegítimo basado en fuertes componentes éticos y morales. ${ }^{10}$

No obstante lo dicho, y por algunos ejemplos que vamos a señalar, la influencia de dicha doctrina es cada vez menor. Así, en nuestra postura, dada la naturaleza propia del arbitraje internacional y la relevancia que supone la autonomía de las partes que mediante el arbitraje deciden dejar de someter la disputa a las cortes nacionales, debe considerarse un enfoque amplio a la hora de analizar aspectos como la admisibilidad o no de los TPF en el ámbito arbitral. Dado que son doctrinas originadas en el área de litigios.

Acceso el 4 de marzo de 2019.

http://www.kluwerarbitration.com/CommonUI/document.aspx?id=kli-ka-joia-330105

${ }^{10}$ De conformidad con Lisa Bench Nieuwveld y Victoria Shannon. 2012. Third-Party

Funding in International Arbitration. Chapter 5. pp. 95 - 116. (Kluwer Law

International 2012). 
El fundamento base de ambas doctrinas es evitar que exista un eventual interés por parte de un tercero en el financiamiento y que esto genere una verdadera industria de arbitrajes dado los beneficios económicos que la actividad reporta. Si bien vemos que esta idea -al menos en lo que refiere al arbitraje- se ha ido disipando, es sin dudas la base de determinados desarrollos efectuados como forma de evitar la utilización de los TPF en el arbitraje con un fin contrario a la ética o la moral, afectando así la transparencia que el arbitraje requiere.

En este sentido, actualmente las cortes de Inglaterra y Hong Kong han establecido que las doctrinas de "champetry and maintenance" no aplican cuando estamos refiriendo a casos frente a Tribunales arbitrales. Claramente la habilitación del uso de TPF en jurisdicciones como la inglesa -donde se originaron las relativas prohibiciones- tiene una importante consecuencia en tanto supone que el fundamento que se encontraba atrás de la misma de una $\mathrm{u}$ otra forma ha desaparecido. ${ }^{11}$

Además, sin perjuicio del avance que se ha producido en la materia, la regulación del fenómeno no ha acompasado su evolución. Así, no existe una regulación internacional y desde el punto de vista regional ha sido regulado únicamente por el AECG entre Canadá y la Unión Europea, el tratado bilateral de inversiones entre la Unión Europea y Vietnam (aun no en vigor) ${ }^{12}$ y el tratado de inversiones entre la Unión Europea y Singapur (pendiente de adopción) $)^{13}$.

${ }^{11}$ Este elemento fue claramente señalado por Lord Neberger ex presidente de la Suprema Corte del Reino Unido al establecer que: “Thus, the public policy rationale regarding maintenance and champerty has turned full circle. Originally their prohibition was justifiable as a means to help secure the development of an inclusive, pluralist society governed by the rule of law. Now, it might be said, the exact reverse of the prohibition is justified for the same reason." Lord Neuberger.2013 "From Barretry, Maintenance and Champerty to Litigation Funding" Harbour Litigation Funding First Annual Lecture. Acceso el 4 de marzo de 2019.

https://www.supremecourt.uk/docs/speech-130508.pdf .

${ }^{12}$ Consejo Europeo, Consejo de la Unión Europea. Acuerdos comerciales de la UE. Acceso el 10 de marzo de 2019. https://www.consilium.europa.eu/es/policies/tradepolicy/trade-agreements/

${ }^{13}$ Consejo Europeo ..., 
Por su parte, algunas jurisdicciones han dado el paso de regular el fenómeno. Así, en el caso de Singapur en donde existía una clara prohibición del uso de TPF el mismo ha sido regulado para permitir su uso en determinadas condiciones estrictamente establecidas. De esta forma, Singapur -uno de los centros de arbitraje más importantes del mundo- reguló la actividad de los TPF bajo una reforma a su Código Civil ${ }^{14}$, disponiendo que para que la actividad desarrollada por el TPF sea legítima, la misma debe ser ejecutada por profesionales en el área, entre otras condiciones.

Por tanto, en el régimen de Singapur, todos los casos que queden fuera del marco regulatorio se encuentran estrictamente prohibidos. Esto es una clara consecuencia de las doctrinas de "champetry and maintenance" que claramente continúan influenciado en el tema objeto de estudio. Es más, la propia regulación establece la prohibición de ejecutar los acuerdos de financiamiento cuando los mismos no cumplen con las condiciones legalmente dispuestas. Por el contrario, en dichas circunstancias, el TPF no puede liberarse de su obligación de abonar el financiamiento acordado. ${ }^{15}$

Dicha regulación tiene como lógica penalizar únicamente al financiador -en tanto se lo presume mayormente capaz de conocer los riesgos por el incumplimiento efectuado- que a la parte financiada. Supone además un incentivo de incumplir con la normativa para ambas partes. Ahora, si nos ponemos a pensar que tanto el TPF como la parte están debidamente en conocimiento y asesoradas en lo general por firmas jurídicas que conoce el régimen aplicable, las consecuencias deberían en el caso ser similares para ambas partes.

\section{3) La regulación actual de los TPF}

\footnotetext{
${ }^{14}$ De conformidad con Jonathan Choo, y YiHui Teo.2017: 'The Next Chapter in the Evolution of Singapore's Legal Landscape: 3rd Party Funding' Acceso el 5 de marzo de 2019. <https://www.twobirds.com/en/news/articles/2017/singapore/the-next-chapter-inthe-evolution-of-singapores-legal-landscape-3rd-party-funding.

${ }^{15}$ Choo, Jonathan y YiHui Teo 2017.
} 
Cuando hablamos de regular el régimen aplicable a los TPF, nos referimos en particular a la regulación de la obligación o no de revelar la existencia de un TPF. La razón de ello es uno de los principales temores que existen en el mundo del arbitraje respecto de los TPF que refiere a la posibilidad de que los mismos de una $\mathrm{u}$ otra forma pueden llegar a influenciar negativamente en la imparcialidad e independencia de los árbitros, que es uno de los principales aspectos que hacen a la naturaleza del proceso arbitral.

No existe a nivel internacional, ningún tipo de regulación de los TPF con carácter general. Si desde el punto de vista regional, encontramos la innovadora regulación efectuada por el AECG entre Canadá y la Unión Europea y los demás tratados de inversión antes mencionados.

Pero, como manifestáramos en la introducción, el hecho de que no exista una regulación del fenómeno no puede considerarse que el mismo se encuentre prohibido o no pueda utilizarse, siendo la conclusión totalmente contraria. Esto lleva a establecer que en el mundo actual existen tres tipos de situaciones referidas a lo que es TPF y regulación: (i) Inexistencia de regulación, (ii) Regulación (iii) Prohibición. Naturalmente la situación (iii) es la no querida, dado que consideramos que el TPF es una herramienta fundamental en el desarrollo del arbitraje. Pero tampoco la inexistencia de regulación es la situación ideal y entendemos que se debe dar paso a la tercera situación, esto es, a la búsqueda de una regulación de los TPF.

Naturalmente, estos son aspectos relevantes para analizar dado que deberá estarse a lo que establezca la jurisdicción aplicable tanto al acuerdo de financiamiento (en adelante “Acuerdo de Financiamiento"), así como a la ley de asiento del arbitraje, como a las eventuales jurisdicciones donde el laudo quiera eventualmente ejecutarse.

Las ventajas que implican el uso del TPF ha llevado como vimos a la apertura de jurisdicciones como la inglesa y el innovador enfoque que ha presentado Singapur. Esto es también ha sucedido con jurisdicciones relevantes desde el punto de vista arbitral como son Ginebra y Paris donde se permite el TPF.

También y en la misma forma que lo hiciera Singapur, fue el turno de Hong Kong, mediante una regulación que puso fin desde junio de 2017 a las prohibiciones que existían para el desarrollo de este tipo de financiamiento. La regulación tiene un fuerte componente ético que busca en base a un código de conductas la creación de un marco donde pueda utilizarse el TPF buscando evitar cualquier forma de abuso que pudiera 
generarse por la utilización de los $\mathrm{TPF}^{16}$, que es justamente uno de los mayores temores que existen a su respecto.

Distinto es lo que sucede en las legislaciones de base civilista donde no existen en su mayoría prohibiciones y que son sin lugar a duda un buen lugar donde explotar el uso de los TPF. ${ }^{17}$

Vimos además ejemplos como el AECG entre Canadá y la Unión Europea donde claramente se regula la obligación de revelar la existencia de un TPF, siendo un buen ejemplo para futuras regulaciones.

Por su parte, la complejidad de un sistema federal como el de los Estados Unidos tiene como consecuencia que las regulaciones varían según el estado en el que nos encontremos, elemento a ser considerado por las partes y los financistas. Así, de conformidad con un estudio realizado ${ }^{18}$ en 19 estados $^{19}$ e incluso en el Distrito de Columbia el uso de los TPF es considerado violatorio de la ley, siendo si permitido el uso de los TPF en otros estados.

Continuando con el análisis de la región, la ventaja particular de Latinoamérica es justamente la inexistencia de regulación al respecto y por tanto la inexistencia de prohibición de cualquier tipo. La única regulación que existe en el continente es en Brasil por parte de la Centro de Arbitraje y Mediación de la Cámara de Comercio Brasil Canadá (CAM-CCBC) que ha desarrollado una guía de cómo actuar en presencia de un $\mathrm{TPF}^{20}$

\footnotetext{
${ }^{16}$ Harbour litigation funding. 2017 "Hong Kong formalises third party funding in arbitration" Acceso el 9 de marzo de 2019.

https://www.harbourlitigationfunding.com/hong-kong-formalises-third-party-fundingarbitration/

17 De conformidad con la Profesora Catherine Rogers -quien ha estudiado en forma pormenorizada el fenómeno- los TPF se encuentran prohibidos en Grecia y Portugal y en otros países como Alemania, Suiza y Holanda la actividad se está desarrollando. Catherine Rogers, Ethics in International Arbitration (Oxford, 2004), 190.

${ }^{18}$ Bench Nieuwveld, Lisa y Victoria Shannon ...,

${ }^{19}$ Alabama, Alaska, Connecticut, Delaware, the District of Columbia, Georgia, Illinois, Indiana, Kansas, Kentucky, Minnesota, Mississippi, Nevada, New Mexico, Pennsylvania, Rhode Island, South Dakota, Utah, Vermont, and Wisconsin.

${ }^{20}$ Resolución 18/2016, de 20 de julio de 2016, del Centro de Arbitraje y Mediación de la Cámara de Comercio Brasil Canadá. Acceso el 12 de marzo de 2019.
} 
Otras asociaciones han regulado algunos puntos relativos a los TPF como ser la "Association of Litigation Funders of England and Wales" (Asociación de financiamiento de litigios de Inglaterra y Gales) que en su propio código de conducta se desarrollan elementos para evitar conflictos éticos. Sin perjuicio de que este código no obliga a quienes no son parte de la asociación tiene la ventaja de ser un intento de "autoregulación", por los propios actores involucrados en el tema, ${ }^{21}$ cuya ejemplificación podría seguirse por otros organismos de similar naturaleza

Corresponde además referir a las Directrices IBA sobre Conflictos de Intereses en Arbitraje Internacional 2014" (en adelante "Directrices IBA sobre conflicto"), las cuales representan en el mundo del arbitraje internacional una importante herramienta. La reforma que se efectuó en 2014 de las Directrices IBA sobre conflicto introdujo algunos elementos relativos a los TPF, que sin perjuicio de que debió haber regulado el tema en forma más específica, son un gran avance.

De esta forma, en el marco de las Directrices IBA sobre conflicto la existencia de un TPF puede suponer en el caso una situación de color rojo o naranja, que son aquellas situaciones que en menor (naranja) o mayor medida (rojo) pueden afectar el estándar de independencia e imparcialidad de un árbitro. La intención de las Directrices IBA sobre conflicto es justamente evitar la existencia de cualquier conflicto de interés.

Pero además, las Directrices IBA sobre conflicto cuando enumera distintos principios generales, en el principio número seis "relaciones" incluye específicamente un concepto de TPF. $^{22}$ También se hacen referencias al momento de explicar el principio general de "deberes de las partes y del árbitro", en este sentido, se indica que el deber que tienen las

\section{https://ccbc.org.br/cam-ccbc-centro-arbitragem-mediacao/resolucao-de-}

\section{disputas/resolucoes-administrativas/ra-18-2016-financiamento-de-terceiros-em-}

\section{arbitragens-cam-ccbcl}

${ }^{21}$ La tesis de que habla de la importancia de la auto-regulación fue desarrollada por la propia Catherine Rogers en "Rogers, Ethics in International..."

${ }^{22}$ La definición de las Reglas IBA sobre conflicto menciona lo siguiente: “A estos efectos, los términos 'tercero financiador' y 'aseguradora' se refieren a cualquier persona o entidad que contribuya con fondos, $\mathrm{u}$ otro tipo de apoyo material, al desarrollo del proceso en interés de la demanda o defensa del caso y que tenga un interés económico directo en, o un deber de indemnizar a una parte por, el laudo que se vaya a emitir en el arbitraje." (Pág. 17). 
partes de revelar cualquier relación directa o indirecta entre el árbitro y la parte ha sido extendido a aquellas personas o entidades que tienen un interés económico directo en el laudo a dictarse, ejemplificando a aquellas entidades que deben proveer fondos para el arbitraje o que tienen el deber de indemnizar a cualquiera de las partes ${ }^{23}$.

La incorporación de estos elementos a las Directrices IBA sobre conflicto -sin perjuicio de su naturaleza- son sin dudas relevantes en tanto dichas Directrices suponen un importante instrumento para el arbitraje internacional. Naturalmente se pudo haber regulado otros aspectos del fenómeno del financiamiento, pero esto no resta importancia al avance que dichas directrices suponen para los TPF y su actual desarrollo.

En lo que respecta a las instituciones de arbitraje (CCI, CNUDMI, etc.) la regulación del tema se ha efectuado en forma lenta, pero si existen hoy algunos importantes avances en la materia. Así, encontramos las "Guidance Note on Conflict disclosures by arbitrators" de la CCI que refieren específicamente a los $\mathrm{TPF}^{24}$. También la CCI estudió el fenómeno de los TPF en un reporte relativo a las decisiones respecto en los costos del arbitraje ${ }^{25}$. Por su parte, debemos referir a los actuales esfuerzos de reforma de las reglas de CNUDMI cuya propuesta de modificación de la "Regla 21"26 en sede de constitución del tribunal incluye una específica regulación de los TPF y la necesidad de revelar su existencia. Existiendo además otras instituciones que también han regulado el uso y

${ }^{23}$ Directrices IBA sobre Conflicto de Intereses en el Arbitraje Internacional, 23 de Octubre de 2014 (International Bar Association, Pág. 17).

${ }^{24}$ De conformidad con Aren Goldsmith and Lorenzo Melchionda. 2016. The ICC's

Guidance Note on Disclosure and Third-Party Funding: A Step in the Right Direction"

Kluwer Blog Arbitration. Acceso el 11 de marzo de 2019.

http://arbitrationblog.kluwerarbitration.com/2016/03/14/the-iccs-guidance-note-ondisclosure-and-third-party-funding-a-step-in-the-right-direction/

${ }^{25}$ International Chamber of Commerce, Decisions on Costs in International Arbitration, International Chamber of Commerce Arbitration and ADR Commission Report (ICC, 2015).

${ }^{26}$ CIADI.2018. Propuesta de Enmiendas a las Reglas del CIADI - Documento de Trabajo. ICSID Secretariat. Acceso 10 de marzo de 2019. https://icsid.worldbank.org/en/Documents/Amendments_Vol_Three.pdf 
desarrollo de los TPF. ${ }^{27}$ Debiéndose considerar que las instituciones arbitrales son naturalmente uno de los principales actores en el mundo del arbitraje y la influencia de la actividad que ejercen es vital.

\section{Conflictos de intereses: El gran desafío de los TPF}

\section{1) La supervivencia y desarrollo de los TPF y la influencia de los aspectos éticos}

Uno de los principales desafíos y obstáculos para ser sorteados por los TPF es justamente el que implica demostrar que estos fondos no son un verdadero riesgo para el estándar de independencia e imparcialidad del arbitraje, uno de los principales elementos que deben ser celosamente protegidos en el mundo del arbitraje.

Nadie niega la relevancia que tiene la figura de los TPF en la actualidad y como los mismos han generado diversas discusiones en el mundo del arbitraje. El crecimiento de los TPF y su continuidad en el mundo arbitral va a depender pura y exclusivamente de sortear todos aquellos cuestionamientos que actualmente se le imputan. Y un elemento central puede ser justamente hacer hincapié en las ventajas que el uso de los TPF supone. De esta forma, si las partes tienen la posibilidad de que exista financiamiento de la actividad arbitral, va a ser más probable que opten por el arbitraje como medio de solución de disputas. También que se utilice como forma de reducir los riesgos que conlleva someter una disputa al arbitraje, ya sea por los costos que la misma supone, así como la eventualidad de deber abonar los costos de la otra parte cuando se es condenado a ello. Correspondiendo hacer referencia a la cita ya transcripta en relación con lo resuelto en

${ }^{27}$ Como ser el "Singapore International Arbitration Centre (SIAC)" en su Regla 24 del SIAC Investment Arbitration Rules y el China International Economic and Trade Arbitration Commission (CIETAC) en su International Investment Arbitration Rules (2017) en su Art. 27. De conformidad con CIADI ..., 667. 
Manuel García Armas y otros v. Venezuela ${ }^{28}$ y los motivos que pueden llevar a adoptar por el uso de un TPF.

Ahora, ¿existe el riesgo de que una creciente industria de financiamientos de arbitraje genere procesos arbitrales por los beneficios económicos que la actividad conlleva? Entendemos que la única forma de que esto no suceda es regulando a los TPF, pero de ninguna manera prohibiendo los mismos. No existe además ningún estudio que demuestre tal premisa.

Es por eso por lo que el marco regulatorio de la actividad de los TPF debe seriamente considerar todo el componente ético que influye en la actividad de los TPF y que el objetivo inicial es preservar el estándar de independencia e imparcialidad de los árbitros y como consecuencia la reputación del sistema arbitral en su conjunto.

\section{2) Conflictos de intereses y situaciones de riesgo ante la utilización de los TPF}

Cuidar la existencia de conflictos de intereses es uno de los principales objetivos que se consideran al estar frente a un procedimiento arbitral. La principal garantía del proceso arbitral es justamente un proceso transparente, donde los árbitros carecen de cualquier tipo de interés en el objeto de la disputa y aun en el caso de ser seleccionados por las partes, los mismos actuarán de forma completamente imparcial e independiente. Es la base del arbitraje mismo y es una de las principales razones por la cual las partes deciden excluir las jurisdicciones estatales.

La independencia e imparcialidad son dos elementos estrictamente conectados y que deben visualizarse en su conjunto. Así, conforme enseña la teoría arbitral tradicional, el elemento independencia se observa desde un punto de vista intrínseco y se convierte así en uno de los principales indicadores de si existe o no determinada imparcialidad la cual se observa desde un punto de vista extrínseco.

\footnotetext{
${ }^{28}$ Manuel García Armas y otros v. Venezuela. 20 de junio de 2018. Caso CPA- Corte Permanente de Arbitraje N²016-08.
} 
La búsqueda de proteger dichos valores en su máxima expresión ha llevado a generar distintos mecanismos que permiten detectar la existencia de cualquier tipo de conflicto. Así, las diversas reglas de arbitrajes institucionales establecen mecanismos como forma de evitar la existencia de conflictos, los que básicamente suponen ciertas declaraciones juradas realizadas por los árbitros, su análisis y eventuales consecuencias en caso de detectarse la existencia de algún tipo de conflicto.

\section{3) La obligación de revelar la existencia de un TPF}

Uno de los principales aspectos que se discute en el mundo del arbitraje actual es si debe existir o no la obligación de revelar la existencia de un TPF. Este aspecto se encuentra intrínsecamente vinculado con los eventuales conflictos de intereses que se puedan producir. Debe tenerse en cuenta que un proceso arbitral puede transcurrir sin que ninguna de las partes conozca o descubra la existencia de un TPF. No obstante, no relevar su existencia, no es la situación ideal y puede perfectamente ocurrir que se descubra su existencia ante un problema que se genere, produciéndose importantes consecuencias.

En forma adicional, claramente no alcanza con solo revelar la existencia de un TPF sino que deben revelarse determinados elementos que impliquen justamente conocer aquellos aspectos que pueden en uno u otro caso generar conflictos de intereses, pero en el caso, también cuidar no dar a conocer aquellos elementos que puedan afectar a las partes del Acuerdo de Financiamiento y que no sean datos relevantes para ser conocido ni por los árbitros, ni por las demás partes.

Así, por ejemplo, en Manuel García Armas y otros v. Venezuela ${ }^{29}$ el Tribunal - ante el envió de un Acuerdo de Financiamiento en el que faltaban algunos elementos expresaba:

Preliminarmente, mediante la OP4 y la RP3, el Tribunal determinó que, en su deber de salvaguardar la integridad del procedimiento, los Demandantes debían revelar el texto del Acuerdo de Financiamiento. En cumplimiento de dicha orden, los Demandantes enviaron el texto, pero expurgado, por considerar que ciertas partes

\footnotetext{
${ }^{29}$ Manuel Garcia Armas y otros v. Venezuela. 20 de junio de 2018. Caso CPA- Corte Permanente de Arbitraje N²016-08.
} 
estaban protegidas por principios de privilegio y confidencialidad, adjuntando a su tiempo y según la determinación del Tribunal Arbitral sus anexos (también con expurgaciones). Ello fue respetado por el Tribunal al decidir luego, en su OP5 y RP4, compartir con la Demandada el Acuerdo de Financiamiento junto son sus anexos expurgados tal cual fueron transmitidos por los Demandantes al Tribunal; encontrando así el balance justo entre los intereses legítimos de ambas Partes. ${ }^{30}$

En el mundo del arbitraje actual no existe con aspectos generales una obligación de revelar la existencia de un TPF, lo que no implica en el caso que no sea un elemento en el que la comunidad arbitral debe trabajar. Existen si algunas regulaciones como es el caso del AECG entre Canadá y la Unión Europea que se constituye en la actualidad como una normativa de avanzada, la que como vimos se ha replicado en otros tratados de inversión. También lo hace el Código de Conducta profesional 2015 de Singapur, obligando a las partes a relevar tal existencia.

La evolución del uso de los TPF ha demostrado que, en algunos casos, sin perjuicio de no existir una obligación para ello, si se ha hecho pública su existencia ${ }^{31}$, lo que supone entonces una situación ideal.

A pesar de ello, la regulación de una obligación de revelar la existencia de un TPF es un elemento para continuar trabajando dado que conocer la existencia de un TPF puede evitar cualquier tipo de conflicto de interés, así como cualquier situación que pueda poner en riesgo los estándares de independencia e imparcialidad de los árbitros.

Naturalmente y como hemos manifestados, el TPF es un fenómeno nuevo y no todas las partes se encuentran en conocimiento de si necesitarán utilizar el mismo o no en el futuro. Pero, una buena práctica arbitral, sería incluir en las clausulas arbitrales, que en el caso de que una de las partes eligiera un financiamiento de terceros, tendría que necesariamente revelar su existencia y desde ya determinados elementos del Acuerdo de

${ }^{30}$ Manuel Garcia Armas y otros v. Venezuela. 20 de junio de 2018. Caso CPA- Corte Permanente de Arbitraje $N^{\circ}$ 2016-08

${ }^{31}$ Como por ejemplo el caso de Oxus Gold que hizo pública mediante un comunicado de prensa en relación con la existencia de un TPF, incluyendo algunos términos del acuerdo efectuado. (Conforme Maxi Scherer. 2012. "Out in the open? Third-party funding in arbitration".

Acceso el 5 de marzo de 2019. https://www.cdr-news.com/categories/third-partyfunding/out-in-the-open-third-party-funding-in-arbitration 
Financiamiento. Sin perjuicio de ello, según es de nuestro conocimiento, no es un elemento que suele pactarse en forma previa, aunque sería un elemento positivo, activando un mecanismo solo para aquellos casos en donde definitivamente se utilice este tipo de financiamiento.

Ahora, el hecho de que no exista una regulación expresa en la cláusula arbitral ni tampoco una obligación de hacerlo, no implica que las partes en cumplimiento de estándares éticos que regulan la actividad arbitral igualmente decidan esclarecer la existencia de un TPF. Porque, además, es mucho más beneficioso para las partes esclarecer esa situación. ¿Qué sucedería en el caso de que en forma posterior a emitido el laudo, un eventual problema entre el financiador y la parte, revelara la existencia del TPF y esto tuviera como consecuencia el descubrir un determinado conflicto de interés u otra situación de índole similar? Claramente daría lugar a una solicitud de anulación del laudo con importantes chances de éxito.

Sostener que corresponde revelar la existencia de un TPF, tiene otras consecuencias. La primera de ella es determinar cuáles son los elementos que deben darse a conocer, donde nos encontramos ante una primera discusión que presenta diversas posturas. Debiendo recordar lo mencionado anteriormente con relación a lo dispuesto por el Tribunal en Manuel García Armas y otros v. Venezuela en referencia al balance que debe existir en relación con la información del Acuerdo de Financiamiento que debe aportarse y aquella que puede ser confidencial para las partes que integran el mismo.

Sin perjuicio de que la lógica indica que lo mejor -a los efectos de asegurar el cumplimiento de los estándares de independencia e imparcialidad- sería justamente revelar la existencia de la totalidad del Acuerdo de Financiamiento, no puede dejarse de considerar que determinados aspectos comerciales contenidos de los mismos atentan contra dicho propósito.

Entendemos que el cumplimiento de estándares claros en materia arbitral, llevan en el caso a sostener que si corresponde revelar todos aquellos aspectos que puedan de una $u$ otra manera suponer un conflicto de interés o que sea evidente que pueda causar una situación no querida ni por las partes ni por los árbitros.

Por tanto, ya hablamos de la necesidad de revelar la existencia de un TPF, la necesidad de incluir ciertos aspectos básicos en dicha revelación, lo que nos llega a una tercera interrogante: ¿Cuándo? A falta de regulación y dado los elementos involucrados, una respuesta básica se impone, es decir, “cuanto antes mejor”. Cualquier tipo de conflicto de interés que pueda producirse, cualquier situación que implique en el caso la violación 
de un estándar ético o una prohibición legal, será debidamente prevenida si conocemos de antemano dicha situación.

Así, el AECG entre Canadá y la Unión Europea regula en su art. 8.26 que la obligación de revelar la existencia de un TPF debe realizarse al momento del ingreso de la demanda o en el caso de que el acuerdo sea posterior apenas el mismo sea acordado.

Además, se concluye que si se llegara a regular la obligación de revelar la existencia de un TPF, corresponderá incondicionalmente revelar aquellos elementos que justamente implican una verdadera revelación, adecuada a los estándares que un proceso arbitral requiere.

\section{4) ¿Por qué preocupa conocer la existencia de un TPF?}

¿Es la obligación de revelar la existencia de un TPF un mero capricho de los actores del mundo arbitral ante la existencia de un nuevo actor? Claramente no y tiene sus fundamentos en distintos aspectos que pueden de una $u$ otra manera afectar la imagen de la institución arbitral.

En este sentido, es claro que revelar la existencia de un TPF en forma tardía puede traer consecuencias negativas que pueden suponer en el caso hasta la nulidad del laudo arbitral, elemento que deberá observarse caso a caso. Esto no es favorable para ninguna de las partes involucradas, pero tampoco para el arbitraje como método alternativo de resolución de disputas.

La revelación de la existencia de un TPF sin lugar a duda previene cualquier tipo de situación no querida por el arbitraje y no solo protege a las partes en sí, sino también a los árbitros que de conocer la existencia de un TPF y de un eventual conflicto de interés, deberán revelarlo en forma inmediata para que las partes puedan en forma libre evaluar si esto afecta su independencia e imparcialidad.

Un elemento recurrente en relación con los problemas que puede generar un TPF son los relativos a cuanto puede afectar el mismo en la estrategia que se sigue en el arbitraje y como los mismos pueden llegar a convertirse en el verdadero elemento de control del litigio. También como el desarrollo del fenómeno puede suponer en el caso crear una verdadera industria que tenga como consecuencia la generación de arbitrajes cuando no 
existen verdaderas razones de "justicia" involucradas, pero si un verdadero beneficio económico. En este sentido, el regular determinados elementos como la obligación de revelar la existencia de un TPF deviene relevante.

Claramente, de la misma forma que existen quienes apuestan a promover la obligación de revelar la existencia de un TPF, también encontramos sus detractores. En este sentido, un representante de un TPF ha indicado que aquellos que presentan argumentos en favor de la obligación de revelar la existencia de un TPF demuestran un verdadero desconocimiento de cómo funciona el mecanismo de financiamiento ${ }^{32}$. Opinión que además se basa en determinados informes técnicos, manifestando en defensa de su postura que claramente el Acuerdo de Financiamiento cuida todos aquellos aspectos que puedan suponer un conflicto de intereses y una imposibilidad de ejecutar el laudo, en tanto el mismo traería en definitiva perjuicios económicos para todas las partes involucradas.

Sin perjuicio que lo expresado no supone un pronunciamiento mayoritario, si es un elemento para atender, dado que justamente los que menos interés tienen en que se genere un problema en el reconocimiento o ejecución de determinado laudo es el financista que se beneficia del eventual caso que está financiando. Igualmente, en nuestro entender esto no debe ser óbice para proceder a una regularización, de forma de garantizar determinados elementos propios de la actividad arbitral. Es más, un sistema que regule a los TPF es un elemento a favor de la promoción y desarrollo del fenómeno lo que debe ser debidamente valorado por los actores involucrados.

Pero, además, siendo la actividad de financiamiento lícita y en tanto los financistas no tengan nada para ocultar, no debería existir oposición alguna al momento de crear un mecanismo que genere mayores garantías a las partes y árbitros involucrados en el proceso.

\section{5) ¿Qué elementos y a quién revelar la existencia del TPF?}

${ }^{32}$ Christopher Bogart. 2016 'Third-Party Financing of International Arbitration' Global Arbitration Review. Acceso el 13 de marzo de 2019. http://globalarbitrationreview.com/insight/the-european-arbitration-review$\underline{\text { 2017/1069316/third-party-financing-of-international-arbitration }}$ 
Continuando con el razonamiento que venimos efectuando, entendemos que no quedan dudas respecto de la necesidad de revelar la existencia de un TPF. Ahora, conforme indicáramos con anterioridad, esto no significa de modo alguno que todos los elementos deben ser revelados.

En este sentido, sin perjuicio de las cláusulas que cada documento en particular pueda contener, todo financiamiento empieza con un "Acuerdo de Financiación”, este acuerdo regula todos y cada uno de los aspectos que rigen a la actividad de financiamiento. Su relevancia es mayor aun, cuando no existe una ley que regule este tipo de acuerdo, los que para el derecho civil podrían llegar a considerarse como contratos innominados. Esto supone además que cada jurisdicción aplicará determinadas reglas a estos contratos según al tipo que se adapten, efectuando las correspondientes analogías y la aplicación de normas supletorias.

Así, sin perjuicio de la dificultad que puede suponer regular esta obligación de revelar la existencia de un TPF si es claro que, aunque no olvidando la relevancia del "Acuerdo de Financiación", no será necesario revelar todos los aspectos que regulan al mismo. Sino que simplemente corresponderá revelar aquellos elementos que sean claves para determinar la existencia de cualquier tipo de conflicto de interés, teniéndose presente los elementos ya señalados en referencia a este punto.

Naturalmente que esta posición también ha encontrado resistencia, entre quienes entienden que corresponde siempre revelar la totalidad del acuerdo como forma de evitar el desconocimiento de cualquier elemento que pueda suponer el apartamiento de determinado estándar. A pesar de ello, entendemos que claramente no son todos los elementos que las partes deben conocer como forma de saber si existe o no un eventual conflicto de interés.

Así, siguiendo nuestro enfoque entendemos que será relevante conocer; las partes involucradas en el arbitraje, cual es el límite del mencionado financiamiento y cuál será el verdadero grado de participación del TPF. Esto nos va a permitir conocer aquellos elementos que si tienen una clara influencia en los eventuales conflictos de intereses que se puedan producir.

Definido esto, la historia no termina puesto que también debemos estrictamente analizar a quien corresponde que le relevemos la existencia del TPF. ¿Es suficiente con hacerlo solo respecto del Tribunal Arbitral? Quizás sí, pero entendemos que no es la opción que mejor se adapta a los principios que rigen al arbitraje y que suponen en todo momento un 
sistema donde la transparencia sea una verdadera guía. Por tanto, la vía que otorga mayores garantías en este sentido es justamente revelar la existencia de un TPF ante las demás partes y al Tribunal, indicando los elementos anteriormente mencionados. Comenzar un procedimiento ocultándole elementos a la otra parte no parecería cumplir prima facie con premisas básicas del instituto del arbitraje.

No nos encontramos con la posibilidad de definir en este momento quien tiene o tendrá la posibilidad de ejecutar la respectiva obligación de revelar la existencia del TPF a todas las partes y al Tribunal y este es un elemento que deberá analizarse a la luz de los reglamentos aplicables al arbitraje concreto, así como la ley de anclaje del arbitraje y otros elementos de las respectivas circunstancias, siendo la casuística más que amplia

\section{Conflictos de intereses: ¿Puede el uso de TPF suponer un crecimiento en materia de conflictos de intereses?}

La protección de estándares tan importantes para el arbitraje internacional como es el de independencia e imparcialidad de los árbitros, hace que sea claramente necesario detectar todas aquellas situaciones que puedan en mayor o menor grado afectar dichos estándares. Esto puesto que garantizar la independencia e imparcialidad de los árbitros supone garantizar la reputación del arbitraje como institución.

A esto se le debe adicionar que actualmente, sin perjuicio del crecimiento del arbitraje internacional como método de solución de conflictos, las firmas, árbitros y demás actores arbitrales muchas veces se repiten, lo que hace más proclive detectar situaciones de conflicto. Si a esto le sumamos la aparición de un nuevo actor como son los TPF que vienen a financiar un nuevo número de casos con un potencial de generar mayores conflictos, claramente nos encontramos ante una realidad que debemos atender.

¿Supone la simple existencia de un TPF y su ocultamiento una situación de conflicto de interés? Claramente no. Pero si revelar la existencia de un TPF en las condiciones antes mencionadas es la única garantía de que no exista tal situación.

Scherer entiende que claramente el crecimiento del uso de los TPF y el limitado número de financistas que existe también genera altas probabilidades de que existan situaciones 
de conflicto. ${ }^{33}$ En este sentido, existen otras numerosas situaciones que pueden suponer la existencia de conflictos de interés y que se resolverán en forma efectiva con el solo conocimiento de la existencia de un TPF.

Una de las situaciones de potencial conflicto que generalmente se menciona es que sucede si en determinados arbitrajes financiados por un mismo TPF se designa siempre al mismo árbitro. En este sentido, la única forma de conocer si existe alguna conexión entre el árbitro y el financista o si la respectiva designación se debe a cualidades específicas del árbitro, es mediante el conocimiento de la existencia del TPF y el análisis de la situación concreta.

Pero, además, existen determinados riesgos de ocultar la existencia de un TPF que son muy altos para ser asumidos por las partes y que pueden llevar hasta la nulidad del laudo arbitral en caso de detectarse una verdadera situación de conflicto.

A esto se debe sumar que, sin perjuicio del intento de las partes de ocultar la existencia de un TPF, situaciones anómalas como puede ser un eventual conflicto entre el financista y la parte financiada pueden llevar al conocimiento de la existencia del TPF y eventualmente -como mencionamos- suponer el riesgo de anulación si se detectara alguna situación anómala o contraria a principios del proceso arbitral. Por eso es importante regular el presente aspecto como forma de evitar que se afecte a la propia naturaleza del instituto arbitral.

Es por eso por lo que entendemos, siguiendo a autores como Rogers ${ }^{34}$ que es necesario regular principalmente la obligación de revelar todos aquellos elementos que permitan descubrir la existencia de un eventual conflicto de intereses producto de la utilización de un TPF.

Sin dudas el fenómeno de los TPF creció en forma más rápida de lo que se esperaba y la regulación y experiencia no acompañó dicho crecimiento, lo que debe ser un fuerte argumento en pos de su regulación. No siendo la situación de incertidumbre la ideal cuando nos encontramos ante la existencia de un nuevo fenómeno que puede afectar la propia naturaleza del arbitraje.

\footnotetext{
${ }^{33}$ Maxi Scherer, (2012). Out in the open? Third-party funding in arbitration. https://www.cdr-news.com/categories/arbitration-and-adr/out-in-the-open-third-partyfunding-in-arbitration Último acceso, 02/8/19.

${ }^{34}$ Rogers, Ethics in International ...
} 
Asimismo, sin perjuicio de que hemos señalado la existencia de determinados avances en materia regulatoria, claramente los mismos no han sido suficientes y aún quedan diversos aspectos para continuar trabajando. Tiene que ser igualmente visto con buenos ojos los avances que se han sucedido en regímenes como el de Singapur y Hong Kong que no solo son importantes para el avance de los TPF, sino que también para asegurar la continuidad y crecimiento del arbitraje como método de solución de disputas.

Sin lugar a duda no nos enfrentamos ante una tarea fácil donde quizás crear instrumentos con fuerza obligatoria no sea la mejor opción. Recordemos que como han señalado algunos autores, uno de los problemas principales es que los tribunales arbitrales no tienen jurisdicción sobre estas terceras partes, lo que imposibilita en principio decidir y ejecutar medidas en su contra. ${ }^{35}$ Lo que en su caso hace más eficaz imponer obligaciones a las partes del arbitraje -como lo es la obligación de revelar la existencia de un TPF- donde claramente el poder de los árbitros si es aplicable.

El beneficio del arbitraje ante el crecimiento de los TPF dependerá de varios factores y el primero de ellos es claramente una regulación adecuada del fenómeno. Pero también existen otros elementos relevantes, como ser el rol que tienen las instituciones arbitrales dado que gran parte de los arbitrajes hoy son institucionales.

Incluso existe un autor que ha generado un modelo a ser adoptado por instituciones arbitrales. ${ }^{36}$ Las Instituciones arbitrales que adoptaron y las que adopten determinadas regulaciones al respecto claramente ejercerán una influencia sobre el mundo del arbitraje. Hay que considerar que, de la misma forma que las reglas de las instituciones regulan aspectos relativos a los peritos expertos, que son terceras partes, también podría ocuparse del rol y participación de los TPF.

Corresponderá determinar según las circunstancias cual será el instrumento o la forma que evite que el crecimiento y desarrollo de los TPF produzca algún tipo de consecuencia negativa, sino que sea todo lo contrario. Ahí surge la opción de crear simplemente "recomendaciones" o "modelos" a ser adoptados por las distintas jurisdicciones o por las partes en sus cláusulas arbitrales o la posibilidad de crear normativa que obligue a las

\footnotetext{
${ }^{35}$ Rogers, Ethics in International...

${ }^{36}$ Jennifer A. Trusz, 2013 "Full Disclosure? Conflicts of Interest Arising from ThirdParty Funding in International Commercial Arbitration' en Georgetown Law Journal. Acceso el 12 de marzo de 2019. https://georgetownlawjournal.org/articles/111/fulldisclosure-conflicts-of/pdf
} 
partes intervinientes en los arbitrajes. Lo que insistimos deberá evaluarse según la realidad del arbitraje en la jurisdicción respectiva.

Creemos además que las leyes de arbitraje internacional adoptadas en forma reciente en Uruguay y Argentina claramente podrían haber regulado el fenómeno de los TPF. Pero, sin lugar a duda, dado el avance que supone la adopción de las respectivas leyes de arbitraje internacional, pretender que se regularan los TPF era ya un elemento más que ambicioso.

Por último, evitar el surgimiento de conflictos de intereses ante el crecimiento que suponen los TPF dependerá, del conocimiento que respecto del TPF tengan las partes involucradas en el arbitraje y como en su caso, las mismas partes puedan optar en las respectivas clausulas arbitrales regular la posibilidad de que exista este tipo de financiamiento en la formas y condiciones que entiendan pertinente.

\section{Consideraciones Finales}

El objeto principal del presente trabajo era presentar el nuevo fenómeno de los TPF o financiadores de arbitraje y cuál es su rol en el mundo del arbitraje internacional actual. Centrándonos específicamente en los riesgos que el uso y desarrollo de los TPF presenta en materia de conflictos de intereses.

Naturalmente el fenómeno de los TPF y la novedad que los mismos presentan para el mundo del arbitraje han traído consigo una infinidad de interrogantes respecto de cómo el mismo puede afectar e influir en el mundo del arbitraje internacional actual. La mera posibilidad de que el crecimiento de los TPF atente contra principios básicos del instituto como es la imparcialidad e independencia de los árbitros genera indudablemente cierto temor respecto de las eventuales consecuencias negativas que el crecimiento del fenómeno pueda acarrear.

En nuestra opinión, los TPF son un nuevo actor que ha llegado para quedarse en el mundo del arbitraje y entendemos que lo más beneficioso es buscar los mecanismos que permitan regular ciertos aspectos que permitan conocer la existencia de conflictos de intereses. Es por eso por lo que entendemos que la obligación de revelar la existencia de un TPF debe ser debidamente regulada considerando las circunstancias de cada caso en particular. 
Sin dudas revelar la existencia de un TPF es la herramienta que realmente permitirá conocer la existencia de cualquier tipo de conflicto de intereses. Debiendo esta obligación incluir aquellos elementos que claramente permitan a los árbitros y a las partes involucradas poder conocer la existencia de un eventual conflicto de interés.

Ante la presencia de este nuevo fenómeno, las partes involucradas en el arbitraje deben buscar obtener los principales beneficios que el mismo ofrece y al mismo tiempo proteger a la institución arbitral en tanto un uso incorrecto de los TPF puede justamente suponer riesgos para la confianza y reputación que las partes tienen respecto del arbitraje internacional. Por lo que también el uso de los TPF supone una fuerte influencia en aspectos éticos que influyen en el desarrollo del arbitraje como medio de solución de disputas

El estudio de los TPF deja en claro que el crecimiento de la utilización de estos no ha acompasado su regulación. Esto supone la necesidad de buscar las mejores alternativas para su regulación, con el objetivo de evitar la existencia de situaciones que vayan en contra de los estándares de imparcialidad e independencia de los árbitros y de buscar la mejor forma de beneficiar al arbitraje como mecanismo de resolución de disputas.

Contribución: $100 \%$ del autor

\section{Bibliografía}

Bench Nieuwveld, Lisa y Victoria Shannon, Third-Party Funding in International Arbitration. Kluwer Law International, 2012.

Bogart, Christopher (2016), Third-Party Financing of International Arbitration en Global Arbitration Review. Acceso el 13 de marzo de 2019.

http://globalarbitrationreview.com/insight/the-european-arbitration-review2017/1069316/third-party-financing-of-international-arbitration

Choo, Jonathan y YiHui Teo (2017), The Next Chapter in the Evolution of Singapore's Legal Landscape: 3rd Party Funding. Acceso el 5 de marzo de 2019. $<$ https://www.twobirds.com/en/news/articles/2017/singapore/the-next-chapter-in-theevolution-of-singapores-legal-landscape-3rd-party-funding

CIADI, 2018. Propuesta de Enmiendas a las Reglas del CIADI - Documento de

Trabajo. ICSID Secretariat. Acceso 10 de marzo de 2019.

https://icsid.worldbank.org/en/Documents/Amendments_Vol_Three.pdf 
Santiago Theoduloz, "Third Party Funding"; su relevancia e influencia actual en el mundo del arbitraje internacional. Revista de Derecho № 20 (dic. 2019 ): 159-187

Consejo Europeo, Consejo de la Unión Europea. Acuerdos comerciales de la UE. Acceso el 10 de marzo de 2019. https://www.consilium.europa.eu/es/policies/tradepolicy/trade-agreements/

Derric, Yeoh. (2016). Third Party Funding in International Arbitration: A Slippery Slope or Levelling the Playing Field? Journal of International Arbitration. Acceso el 4 de marzo de 2019.

http://www.kluwerarbitration.com/CommonUI/document.aspx?id=kli-ka-joia-330105 Goldsmith, Aren and Lorenzo Melchionda. The ICC's Guidance Note on Disclosure and Third-Party Funding: A Step in the Right Direction” Kluwer Blog Arbitration, 2016. Acceso el 11 de marzo de 2019.

http://arbitrationblog.kluwerarbitration.com/2016/03/14/the-iccs-guidance-note-ondisclosure-and-third-party-funding-a-step-in-the-right-direction/ Harbour litigation funding (2017), Hong Kong formalises third party funding in arbitration. https://www.harbourlitigationfunding.com/hong-kong-formalises-third-partyfunding-arbitration/Acceso el 9 de marzo de 2019.

International Chamber of Commerce. Decisions on Costs in International Arbitration, International Chamber of Commerce, Arbitration and ADR Commission Report (ICC 2015).

Neuberger, David, From Barretry, Maintenance and Champerty to Litigation Funding en Harbour Litigation Funding First Annual Lecture, 2017. Acceso el 4 de marzo de 2019. https://www.supremecourt.uk/docs/speech-130508.pdf .

Rogers, Catherine y William Park, Third Party Funding in International Arbitration: The ICCA Queen-Mary Task Force en Penn State Law, The Dickinson School of Law Legal Studies Research Paper No 42, 2014.

Rogers, Catherine, Ethics in International Arbitration. Oxford, 2004.

Scherer, Maxi (2012). Out in the open? Third-party funding in arbitration. Acceso el 5 de marzo de 2019. https://www.cdr-news.com/categories/third-party-funding/out-in-theopen-third-party-funding-in-arbitration

Trusz, Jennifer A. (2013), Full Disclosure? Conflicts of Interest Arising from ThirdParty Funding in International Commercial Arbitration en Georgetown Law Journal. Acceso el 12 de marzo de 2019. https://georgetownlawjournal.org/articles/111/fulldisclosure-conflicts-of/pdf

\section{Jurisprudencia Citada}


Manuel Garcia Armas y otros v. Venezuela. 20 de junio de 2018. Caso CPA- Corte

Permanente de Arbitraje N²016-08.

Philips Morris vs. Uruguay. 8 de Julio de 2016 (envío a las partes), Caso CIADI- Centro Internacional de Arreglo de Diferencias Relativas a Inversiones. CN. ${ }^{\circ} \mathrm{ARB} / 10 / 7$.

\section{Normativa Citada}

Acuerdo Económico y Comercial Global entre Canadá y la Unión Europea

Directrices IBA sobre Conflicto de Intereses en el Arbitraje Internacional, 23 de

Octubre de 2014 (International Bar Association, Pág. 17).

Ley 19.636, Publicación 26 de julio de 2018, Poder Legislativo, Uruguay.

Ley 27.449, Publicación 26/7/2018, Congreso Argentino

Resolución 18/2016, de 20 de julio de 2016, del Centro de Arbitraje y Mediación de la

Cámara de Comercio Brasil Canadá. Acceso el 12 de marzo de 2019. 